HIP-2008-33/TH

\title{
Correlations between sneutrino oscillations and signatures at the LHC in anomaly-mediated supersymmetry breaking
}

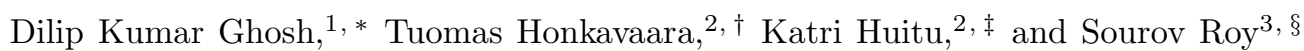 \\ ${ }^{1}$ Theoretical Physics Division, Physical Research Laboratory, Navrangpura, Ahmedabad 380 009, India \\ ${ }^{2}$ Department of Physics, and Helsinki Institute of Physics, \\ P.O.Box 64, FIN-00014 University of Helsinki, Finland \\ ${ }^{3}$ Department of Theoretical Physics and Centre for Theoretical Sciences, \\ Indian Association for the Cultivation of Science, \\ $2 A \& 2 B$ Raja S.C. Mullick Road, Kolkata 700 032, India
}

(Dated: November 17, 2018)

\begin{abstract}
Sneutrino-antisneutrino oscillation can be observed at the LHC by studying a charge asymmetry of the leptons in the final states. We demonstrate this in the context of an anomaly-mediated supersymmetry breaking model which can give rise to a large oscillation probability. The preferred region of the parameter space is characterized by the presence of a sneutrino next-to-lightest supersymmetric particle and a stau lightest supersymmetric particle. We show that the signals studied here have certain correlations with the pattern of the sneutrino oscillation.
\end{abstract}

PACS numbers: 12.60.Jv, 14.60.Pq, 14.80.Ly

Sneutrino-antisneutrino mixing occurs in any supersymmetric (SUSY) model where neutrinos have nonzero Majorana masses. Such $\Delta L=2$ Majorana neutrino mass terms can induce a mass splitting $\left(\Delta m_{\tilde{\nu}}\right)$ between the physical states. The effect of this mass splitting is to induce sneutrino-antisneutrino oscillations [1, 2]. This can lead to the sneutrino decaying into a final state with a "wrong-sign charged lepton," and the lepton number can be tagged in sneutrino decays by the charge of the final state lepton. Here, we assume that the sneutrino flavor oscillation is absent and lepton flavor is conserved in the decay of sneutrino/antisneutrino.

As discussed in [3], the probability of finding a wrongsign charged lepton in the decay of a sneutrino should be the time-integrated one and is given by

$$
P\left(\tilde{\nu} \rightarrow \ell^{+}\right)=\frac{x_{\tilde{\nu}}^{2}}{2\left(1+x_{\tilde{\nu}}^{2}\right)} \mathcal{B}_{\tilde{\nu}^{*}}\left(\tilde{\nu}^{*} \rightarrow \ell^{+} X\right)
$$

where the quantity $x_{\tilde{\nu}}$ is defined as $x_{\tilde{\nu}} \equiv \Delta m_{\tilde{\nu}} / \Gamma_{\tilde{\nu}}$, and $\mathcal{B}_{\tilde{\nu}^{*}}$ is the branching ratio for $\tilde{\nu}^{*} \rightarrow \ell^{+}$. This signal can be observed from the single production of a sneutrino at the LHC, provided $x_{\tilde{\nu}} \sim 1$ and $\mathcal{B}_{\tilde{\nu}^{*}}$ is significant.

In this paper, we demonstrate that a particular SUSY mass spectrum possible within the framework of an anomaly-mediated supersymmetry breaking model (AMSB) [4] can lead to such a signature at the LHC. It is evident from the above discussion that the probability of the sneutrino-antisneutrino oscillation depends

ฯAfter 26 February 2009, permanent institute is ${ }^{3}$. crucially on $\Delta m_{\tilde{\nu}}$ and $\Gamma_{\tilde{\nu}}$. If $m_{\nu} \sim 0.1 \mathrm{eV}$, the radiative corrections to the $m_{\nu}$ induced by $\Delta m_{\tilde{\nu}}$ face the bound [2] $\Delta m_{\tilde{\nu}} / m_{\nu} \lesssim \mathcal{O}(4 \pi / \alpha)$, implying $\Delta m_{\tilde{\nu}} \lesssim 0.1 \mathrm{keV}$. Thus, in order to get $x_{\tilde{\nu}} \sim 1$, one also needs the sneutrino decay width $\Gamma_{\tilde{\nu}}$ to be $\sim \Delta m_{\tilde{\nu}}$. Because of the smallness of $\Gamma_{\tilde{\nu}}$, the sneutrino's lifetime would be large enough for sneutrino oscillation to take place before its decay.

However, for a spectrum where $\tilde{\chi}_{1}^{0}$ is the lightest supersymmetric particle (LSP), $\Gamma_{\tilde{\nu}}$ would not be $\lesssim \mathcal{O}(1) \mathrm{keV}$ because of the presence of two-body decays: $\tilde{\nu} \rightarrow \nu \tilde{\chi}^{0}$ and $\tilde{\nu} \rightarrow \ell^{-} \tilde{\chi}^{+}$. If, instead, the mass spectrum is such that

$$
m_{\tilde{\tau}_{1}}<m_{\tilde{\nu}}<m_{\tilde{\chi}_{1}^{0}}, m_{\tilde{\chi}_{1}^{ \pm}},
$$

where the lighter stau $\left(\tilde{\tau}_{1}\right)$ is the LSP, these two-body decay modes are forbidden and the three-body decay modes such as $\tilde{\nu} \rightarrow \ell^{-} \tilde{\tau}_{1}^{+} \nu_{\tau}$ and $\tilde{\nu} \rightarrow \nu \tilde{\tau}_{1}^{ \pm} \tau^{\mp}$ are the available ones. In addition, the branching fraction of $\tilde{\nu}^{*} \rightarrow \ell^{+} \tilde{\tau}_{1}^{-} \bar{\nu}_{\tau}$ final state gives the wrong-sign charged lepton signal. However, having $\tilde{\tau}_{1}$ as a stable charged particle is strongly disfavored by astrophysical grounds. This can be avoided, for example, if a very small $R$-parityviolating coupling $\left(\lesssim 10^{-8}\right)$ induces the decay $\tilde{\tau}_{1} \rightarrow \ell \nu$, which occurs outside the detector after producing a heavily ionized charged track in the detector.

The required spectrum (2) can be obtained in some region of the AMSB parameter space with $\Delta m_{\tilde{\nu}} \lesssim$ $\mathcal{O}\left(4 \pi m_{\nu} / \alpha\right)$. In our analysis, we have $m_{\nu_{i}} \lesssim 0.3 \mathrm{eV}$ $(i=e, \mu, \tau)$. In Fig. 1 we display the region of the parameter space in $m_{0}-m_{3 / 2}$ plane with $\operatorname{sign}(\mu)<0$ and $\tan \beta=6$, where the above spectrum is valid. It is worthwhile to mention that the three sneutrinos are almost mass degenerate ( $\tilde{\nu}_{\tau}$ is slightly lighter though) and 


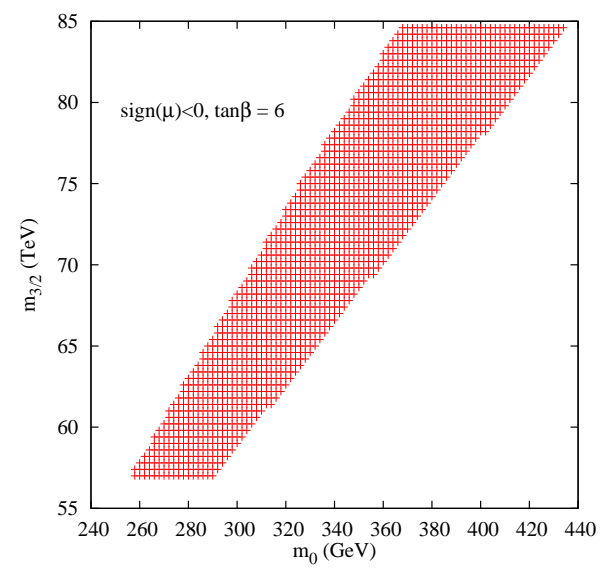

FIG. 1: Parameter space of the AMSB model with sneutrino $\mathrm{NLSP}$ and $\tilde{\tau}_{1} \mathrm{LSP}$ and $m_{\tilde{\ell}_{1,2}}<m_{\tilde{\chi}_{1}^{0}, \tilde{\chi}_{1}^{ \pm}}(\ell=e, \mu)$.

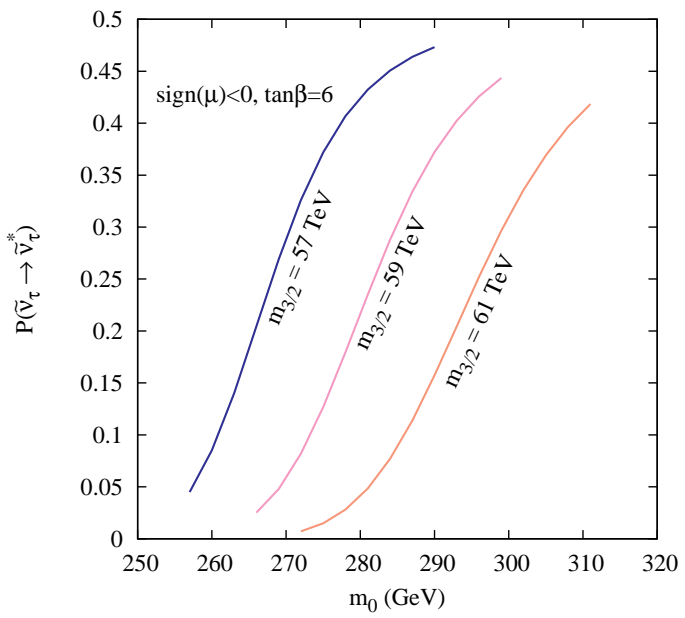

FIG. 2: $\tilde{\nu}_{\tau}$ oscillation probability as a function of $m_{0}$.

they are the NLSP. In this parameter space, $\tilde{\chi}_{1}^{0}$ and $\tilde{\chi}_{1}^{ \pm}$ are heavier than the other charged sleptons.

In Fig. 2, we plot the $\tilde{\nu}_{\tau}$ oscillation probability as a function of the common scalar mass $m_{0}$ for three different choices of $m_{3 / 2}$ with $\operatorname{sign}(\mu)<0$ and $\tan \beta=6$ in the allowed parameter space. It can be seen from this figure that the probability of oscillation can go as high as 0.45 , and it is this probability that we propose to measure. Hence, the AMSB has a good potential to produce signals of sneutrino-antisneutrino oscillation, which can be tested in colliders. This has been noticed earlier; see [3, 5].

Let us remark that the signatures of sneutrino oscillation at the future $e^{+} e^{-}$and $e^{-} \gamma$ linear colliders have been studied in Refs. [3, 5], whereas authors in Ref. [6] discussed sneutrino oscillation in the seesaw extended minimal supersymmetric standard model (MSSM). In this work, the first production process we will consider is

$$
p p \rightarrow \tilde{\nu}_{\tau} \tilde{\tau}_{1}^{+} .
$$

Since $\tilde{\nu}_{\tau}$ decaying to a three-body final state with $\tau^{ \pm}$is difficult to identify due to the small branching ratio and $\tau$ detection efficiency, we look at other channels mediated by virtual $W^{-}$and $H^{-}$. If the $\tilde{\nu}_{\tau}$ oscillates into a $\tilde{\nu}_{\tau}^{*}$, we can have a three-body final state, $\tilde{\nu}_{\tau} \rightarrow \tilde{\nu}_{\tau}^{*} \rightarrow \ell^{-} \tilde{\tau}_{1}^{+} \bar{\nu}_{\ell}$ leading to $\ell^{-} \tilde{\tau}_{1}^{+} \tilde{\tau}_{1}^{+}+\not \not_{T}$ signature from the process in Eq. (3). Here, $\ell=e, \mu$. The cross section for this process is given by $\sigma_{\text {osc }}^{1}=\sigma\left(p p \rightarrow \tilde{\nu}_{\tau} \tilde{\tau}_{1}^{+}\right) \times P_{\tilde{\nu}_{\tau} \rightarrow \tilde{\nu}_{\tau}^{*}} \times \mathcal{B}_{\tilde{\nu}^{*}}\left(\tilde{\nu}_{\tau}^{*} \rightarrow\right.$ $\left.\ell^{-} \tilde{\tau}_{1}^{+} \bar{\nu}_{\ell}\right)$, where $P_{\tilde{\nu}_{\tau} \rightarrow \tilde{\nu}_{\tau}^{*}}$ denotes the sneutrino oscillation probability. When the $\tilde{\nu}_{\tau}$ survives as a $\tilde{\nu}_{\tau}$, one of the possible three-body decays of the $\tilde{\nu}_{\tau}$ is $\tilde{\nu}_{\tau} \rightarrow \ell^{+} \tilde{\tau}_{1}^{-} \nu_{\ell}$. This would lead to $\ell^{+} \tilde{\tau}_{1}^{-} \tilde{\tau}_{1}^{+}+\not p_{T}$ signature from the same process (3). Similar to the oscillation case, the cross section for this process is given by $\sigma_{\text {no osc }}^{1}=\sigma(p p \rightarrow$ $\left.\tilde{\nu}_{\tau} \tilde{\tau}_{1}^{+}\right) \times P_{\tilde{\nu}_{\tau} \rightarrow \tilde{\nu}_{\tau}} \times \mathcal{B}_{\tilde{\nu}}\left(\tilde{\nu}_{\tau} \rightarrow \ell^{+} \tilde{\tau}_{1}^{-} \nu_{\ell}\right)$, where $P_{\tilde{\nu}_{\tau} \rightarrow \tilde{\nu}_{\tau}}$ denotes the sneutrino survival probability.

As mentioned earlier, in the presence of a very small $R$ parity violating coupling, both of these staus would decay outside the detector after traversing the whole length of the detector producing heavily ionized charged track. In this case, the lepton number can be tagged by the charge of the long-lived stau.

Let us define a charge asymmetry parameter in terms of the signal from sneutrino oscillation and no-oscillation:

$$
A_{\mathrm{asym}} \equiv \frac{\sigma\left(\ell^{-} \tilde{\tau}_{1}^{+} \tilde{\tau}_{1}^{+}+\not p_{T}\right)-\sigma\left(\ell^{+} \tilde{\tau}_{1}^{-} \tilde{\tau}_{1}^{+}+\not \phi_{T}\right)}{\sigma\left(\ell^{-} \tilde{\tau}_{1}^{+} \tilde{\tau}_{1}^{+}+\not p_{T}\right)+\sigma\left(\ell^{+} \tilde{\tau}_{1}^{-} \tilde{\tau}_{1}^{+}+\not \not_{T}\right)}
$$

Since $\mathcal{B}_{\tilde{\nu}}\left(\tilde{\nu}_{\tau} \rightarrow \ell^{+} \tilde{\tau}_{1}^{-} \nu_{\ell}\right)=\mathcal{B}_{\tilde{\nu}^{*}}\left(\tilde{\nu}_{\tau}^{*} \rightarrow \ell^{-} \tilde{\tau}_{1}^{+} \bar{\nu}_{\ell}\right)$, one can rewrite Eq. (4) in the following form

$$
A_{\mathrm{asym}}=P_{\tilde{\nu}_{\tau} \rightarrow \tilde{\nu}_{\tau}^{*}}-P_{\tilde{\nu}_{\tau} \rightarrow \tilde{\nu}_{\tau}} .
$$

It is evident from Eq. (5) that $A_{\text {asym }}=-1$ corresponds to no sneutrino oscillation. Hence, any deviation of $A_{\text {asym }}$ from - 1 is the smoking gun signature of sneutrino oscillation. From Eq. (5), it is clear that the measurement of the asymmetry immediately tells us about the sneutrino-antisneutrino oscillation probability $P_{\tilde{\nu}_{\tau} \rightarrow \tilde{\nu}_{\tau}^{*}}$ which is a function of the ratio $x_{\tilde{\nu}} \equiv \Delta m_{\tilde{\nu}} / \Gamma_{\tilde{\nu}}$. Hence, once the total sneutrino decay width is known, one can easily calculate the mass splitting between the sneutrino and the antisneutrino. This mass splitting is proportional to the neutrino mass, and, thus, the measurement of the asymmetry gives us the absolute value of the neutrino mass. This is an alternative way to the neutrinoless double beta decay experiments to probe the absolute value of the neutrino mass. Let us note that there is very little SM background to these signals assuming that the long-lived staus produce heavily ionized charged tracks which can be distinguished from the muon tracks. This is possible, since the staus are much slower than the muons because of their large masses. 
However, there are several other SUSY processes which can give rise to the same final state as our signal. Part of these processes has a sneutrino in the final state and the other part is without a sneutrino. These processes are

$$
p p \rightarrow \tilde{\nu}_{\ell} \tilde{\ell}_{L}^{+} \text {with } \ell=e, \mu, \quad \text { and } \quad p p \rightarrow \tilde{\chi}_{1}^{0} \tilde{\chi}_{1}^{+} .
$$

Here, the relevant decay modes, which can lead to the same signal as in Eq. (3), are for $\tilde{\chi}_{1}^{0}, \tilde{\chi}_{1}^{+}$, and $\tilde{\ell}_{L}^{+}$: $\tilde{\chi}_{1}^{0} \rightarrow \tilde{\nu}_{\ell} \bar{\nu}_{\ell}, \tilde{\nu}_{\ell}^{*} \nu_{\ell}, \tilde{\nu}_{\tau} \bar{\nu}_{\tau}, \tilde{\nu}_{\tau}^{*} \nu_{\tau}, \tilde{\chi}_{1}^{+} \rightarrow \tilde{\ell}_{L}^{+} \nu_{\ell}, \tilde{\tau}_{1}^{+} \nu_{\tau}, \tilde{\ell}_{L}^{+} \rightarrow$ $\tilde{\tau}_{1}^{+} \nu_{\tau} \bar{\nu}_{\ell}$, where $\ell=e$ or $\mu$. Since the charged sleptons are lighter than the neutralinos and the charginos, they decay to three bodies. If $\tilde{\nu}_{\ell}$ oscillates, it will decay to a wrong-sign charged lepton. The relevant $\tilde{\nu}_{\ell}$ decays are $\tilde{\nu}_{\ell} \rightarrow \ell^{+} \tilde{\tau}_{1}^{-} \bar{\nu}_{\tau} \quad$ (with oscillation) and $\tilde{\nu}_{\ell} \rightarrow$ $\ell^{-} \tilde{\tau}_{1}^{+} \nu_{\tau} \quad$ (without oscillation) and correspondingly for $\tilde{\nu}_{\ell}^{*}$. The relevant decays of $\tilde{\nu}_{\tau}$ and $\tilde{\nu}_{\tau}^{*}$ have been discussed earlier.

Thus, for example, $p p \rightarrow \tilde{\chi}_{1}^{0} \tilde{\chi}_{1}^{+} \rightarrow\left(\tilde{\nu}_{\ell} \bar{\nu}_{\ell}\right)\left(\tilde{\ell}_{L}^{+} \nu_{\ell}\right)$ can give rise to the signal $p p \rightarrow \ell^{-} \tilde{\tau}_{1}^{+} \tilde{\tau}_{1}^{+}+\not \phi_{T}$ without $\tilde{\nu}_{\ell}$ oscillation, whereas the same decay chain can also produce $p p \rightarrow \ell^{+} \tilde{\tau}_{1}^{-} \tilde{\tau}_{1}^{+}+\not \phi_{T}$ for oscillating $\tilde{\nu}_{\ell}$. Similarly, $p p \rightarrow \tilde{\nu}_{\ell} \tilde{\ell}_{L}^{+}$leads to $\ell^{+} \tilde{\tau}_{1}^{-} \tilde{\tau}_{1}^{+}+\not p_{T}$ with the oscillation of the $\tilde{\nu}_{\ell}$ into a $\tilde{\nu}_{\ell}^{*}$. The same production process gives rise to the final state $\ell^{-} \tilde{\tau}_{1}^{+} \tilde{\tau}_{1}^{+}+\not \phi_{T}$ when $\tilde{\nu}_{\ell}$ does not oscillate. $\tilde{\chi}_{1}^{0}$ can also decay to a tau-sneutrino and a tauneutrino, leading to the final states $p p \rightarrow \ell^{+} \tilde{\tau}_{1}^{-} \tilde{\tau}_{1}^{+}+\not \phi_{T}$ and $p p \rightarrow \ell^{-} \tilde{\tau}_{1}^{+} \tilde{\tau}_{1}^{+}+\not \phi_{T}$. Heavier neutralinos and chargino are not considered in the production process because of their much smaller cross sections.

The signal can also result from $\tilde{\chi}_{1}^{0}$ cascade decay without any intermediate sneutrinos. For example, $p p \rightarrow$ $\tilde{\chi}_{1}^{0} \tilde{\chi}_{1}^{+} \rightarrow\left(\tau^{-} \tilde{\tau}_{1}^{+}\right)\left(\tilde{\tau}_{1}^{+} \nu_{\tau}\right)$ can lead to $\ell^{-} \tilde{\tau}_{1}^{+} \tilde{\tau}_{1}^{+}+\not p_{T}$, where $\tau^{-}$decays leptonically. Similarly, $p p \rightarrow \tilde{\chi}_{1}^{0} \tilde{\chi}_{1}^{+} \rightarrow$ $\left(\ell^{+} \tilde{\ell}_{L}^{-}\right)\left(\tilde{\tau}_{1}^{+} \nu_{\tau}\right)$ can produce $\ell^{+} \tilde{\tau}_{1}^{-} \tilde{\tau}_{1}^{+}+\not \phi_{T}$.

All the processes discussed above can be considered as the SUSY backgrounds to the signals originating from the process (3). The charge asymmetry $A_{\text {asym }}$ must be considered after taking into account these backgrounds in order to observe the signature of $\tilde{\nu}_{\tau}$ oscillation. One should note that processes involving $\tilde{\nu}_{\ell} / \tilde{\nu}_{\ell}^{*}$ contribute to the final states $\ell^{+} \tilde{\tau}_{1}^{-} \tilde{\tau}_{1}^{+}+\not \not_{T}$ and $\ell^{-} \tilde{\tau}_{1}^{+} \tilde{\tau}_{1}^{+}+\not \phi_{T}$ in an opposite manner compared to the signal processes. Hence, it is indispensable to find suitable kinematical cuts to remove these backgrounds so that the signatures of $\tilde{\nu}_{\tau}$ oscillation can be observed through the measurements of the lepton charge asymmetry defined in Eq. (4).

Nevertheless, we observe that the distributions of two kinematical quantities, namely, the transverse momentum $\left(p_{T}\right)$ of the staus and the missing transverse momentum $\left(\not \not_{T}\right)$ are very crucial in distinguishing these SUSY background processes from the signals. It turns out that, using suitable cuts, one can have $S / \sqrt{B} \approx 5$ or higher even with an integrated luminosity of $30 \mathrm{fb}^{-1}$.

We select the signal events with the following criteria: 1) $\left.\left.p_{T}^{\ell^{ \pm}}>5 \mathrm{GeV}, 2\right)\left|\eta^{\ell^{ \pm}, \tilde{\tau}_{1}}\right|<2.5,3\right)$ transverse momentum of both $\tilde{\tau}_{1}^{-}$'s must satisfy $p_{T}^{\tilde{\tau}_{1}}>100 \mathrm{GeV}$, and 4 )

\begin{tabular}{|c|c|c|c|c|}
\hline \multirow{2}{*}{ Process } & \multicolumn{4}{|c|}{ Cross sections in fb } \\
\cline { 2 - 5 } & $e^{-} \tilde{\tau}_{1}^{+} \tilde{\tau}_{1}^{+} \not_{T}^{\prime}$ & $\mu^{-} \tilde{\tau}_{1}^{+} \tilde{\tau}_{1}^{+} \not_{T}^{\prime}$ & $\mu^{+} \tilde{\tau}_{1}^{-} \tilde{\tau}_{1}^{+} p_{T}^{\prime}$ & $e^{+} \tilde{\tau}_{1}^{-} \tilde{\tau}_{1}^{+} \not_{T}^{\prime}$ \\
\hline$p p \rightarrow \tilde{\nu}_{\tau} \tilde{\tau}_{1}^{+}$ & 1.259 & 1.259 & 3.095 & 3.095 \\
\hline$p p \rightarrow \tilde{\nu}_{\ell} \ell_{L}^{+}$ & 0.373 & 0.373 & 0.303 & 0.303 \\
\hline $\begin{array}{c}p p \rightarrow \tilde{\chi}_{1}^{0} \tilde{\chi}_{1}^{+} \\
\left(\text {with } \tilde{\nu}^{\prime} \text { 's) }\right.\end{array}$ & 0.206 & 0.206 & 0.206 & 0.206 \\
\hline $\begin{array}{c}p p \rightarrow \tilde{\chi}_{1}^{0} \tilde{\chi}_{1}^{+} \\
\left(\text {no } \tilde{\nu}^{\prime} \text { 's) }\right.\end{array}$ & 0.036 & 0.036 & 0.036 & 0.036 \\
\hline
\end{tabular}

TABLE I: Cross sections for $p p \rightarrow \ell^{-} \tilde{\tau}_{1}^{+} \tilde{\tau}_{1}^{+}+\not \not_{T}$ and $p p \rightarrow$ $\ell^{+} \tilde{\tau}_{1}^{-} \tilde{\tau}_{1}^{+}+\not \not_{T}$ from different two-body production processes. Here, $\tan \beta=6, \mu<0, m_{0}=270 \mathrm{GeV}$, and $m_{3 / 2}=57 \mathrm{TeV}$.

$\not \not_{T}<20 \mathrm{GeV}$. The last mentioned two cuts are crucial in clearly identifying signals from the SUSY background.

In Table \, we listed the cross sections of final states described above originating from different $2 \rightarrow 2$ production processes for a particular parameter point, namely, $\tan \beta=6, \mu<0$, and $m_{0}=270 \mathrm{GeV}$, and $m_{3 / 2}=57 \mathrm{TeV}$. These numbers are obtained after applying the selection cuts. One can see that the SUSY background events are well suppressed compared to the signal events. Now, if we calculate the asymmetry, as defined in Eq. (4), from the numbers in the first row of Table I the asymmetry comes out to be -0.42 . If we solve from this for the $\tilde{\nu}_{\tau}$ oscillation probability using Eq. (15), we get $P_{\tilde{\nu}_{\tau} \rightarrow \tilde{\nu}_{\tau}^{*}}=$ 0.29 , which can be compared with Fig. (2). However, as discussed, the SUSY background leads to the same final states and has to be taken into account when calculating the asymmetry.

It should be kept in mind though that, in this situation, there are two different oscillation probabilities involved. One is the tau-sneutrino oscillation probability and the other is the electron (muon) sneutrino oscillation probability. Hence, by measuring the asymmetry as defined in Eq. (4), it would not be possible to measure these two oscillation probabilities. In any case, the measurement of the asymmetry can clearly indicate whether the sneutrinos are oscillating or not. For example, let us again look at the parameter point studied in Table @ and assume that the SUSY background contamination is not possible to be better separated from the signal. In such a situation, the resultant asymmetry is -0.33 when the sneutrinos are oscillating and -0.68 when there is no sneutrino oscillation. Compared to the ideal case with no SUSY background, the measurable asymmetry in the sneutrino oscillation case changes by less than $15 \%$.

In Table II. we show the asymmetries including the SUSY background for three different parameter choices. In all of these cases, the oscillation probability is more than 0.15 . If the parameter set is already known from the measurements of the SUSY spectrum, the expectation for the asymmetry is also known. It is seen from the table that, already with $30 \mathrm{fb}^{-1}$, one can distinguish between the oscillation and no-oscillation cases in these sample points. When $\tan \beta$ grows, the ratio between the SUSY 


\begin{tabular}{|c|c|c|c|c|}
\hline \multirow{2}{*}{$\begin{array}{l}\text { Parameter point } \\
\tan \beta, m_{0}(\mathrm{GeV}), \\
m_{3 / 2}(\mathrm{TeV})\end{array}$} & \multirow{2}{*}{$\begin{array}{c}A_{\text {asym }} \\
\text { osc. } \\
\text { (no osc.) }\end{array}$} & \multicolumn{3}{|c|}{ \pm Errors } \\
\hline & & $30 \mathrm{fb}^{-1}$ & $100 \mathrm{fb}^{-1}$ & $300 \mathrm{fb}^{-1}$ \\
\hline $5,370,81, \mu<0$ & $\begin{array}{c}-0.515 \\
(-0.859)\end{array}$ & $\begin{array}{c}0.072 \\
(0.043)\end{array}$ & $\begin{array}{c}0.040 \\
(0.024)\end{array}$ & $\begin{array}{c}0.023 \\
(0.014)\end{array}$ \\
\hline $6,270,57, \mu<0$ & $\begin{array}{c}-0.325 \\
(-0.676)\end{array}$ & $\begin{array}{c}0.052 \\
(0.041)\end{array}$ & $\begin{array}{c}0.029 \\
(0.022)\end{array}$ & $\begin{array}{c}0.017 \\
(0.013)\end{array}$ \\
\hline $7,248,49, \mu<0$ & $\begin{array}{c}-0.149 \\
(-0.266)\end{array}$ & $\begin{array}{c}0.044 \\
(0.043)\end{array}$ & $\begin{array}{c}0.024 \\
(0.024)\end{array}$ & $\begin{array}{c}0.014 \\
(0.014)\end{array}$ \\
\hline
\end{tabular}

TABLE II: Asymmetries and the corresponding errors for different parameter points. Numbers in the brackets are for the no-oscillation case.

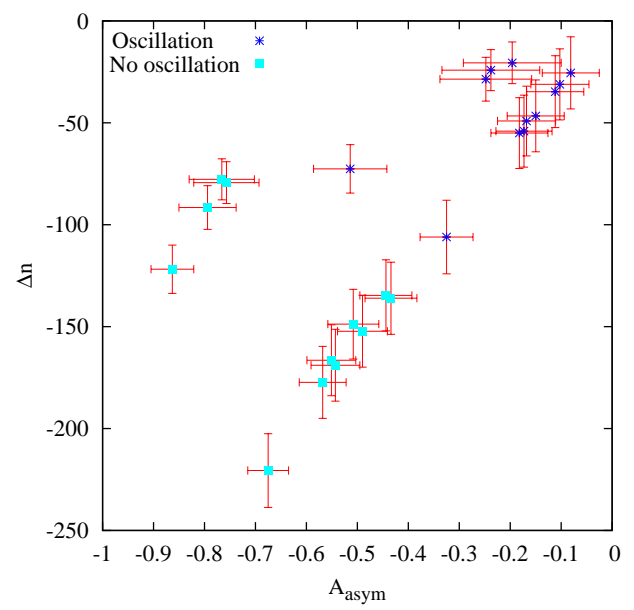

FIG. 3: Correlation between $\Delta n$ and $A_{\text {asym }}$ for the oscillation and the no-oscillation cases for different parameter points.

signal and the background reduces. Thus, this measurement, with the cuts used, is possible for small $\tan \beta$. We find relevant parameter sets for $\tan \beta=5,6,7$, which have been shown in Table

If the SUSY spectrum is not known, one can still deduce in favorable cases whether there is sneutrino oscillation or not. We demonstrate this in Fig. 3 for $\tan \beta=5,6$ and the values of $m_{0}$ and $m_{3 / 2}$ for which the signal cross sections are large. Here, it has been required that oscillation probability is more than 0.25 and $S / \sqrt{B} \gtrsim 5$. We plot the difference $(\Delta n)$ between the numbers of events for $p p \rightarrow \ell^{-} \tilde{\tau}_{1}^{+} \tilde{\tau}_{1}^{+}+\not p_{T}$ and $p p \rightarrow \ell^{+} \tilde{\tau}_{1}^{-} \tilde{\tau}_{1}^{+}+\not p_{T}$ for integrated luminosity $30 \mathrm{fb}^{-1}$ vs the asymmetry. The corresponding errors are shown at the $1 \sigma$ level. One can see from this correlation plot that the sneutrino oscillation represents bigger asymmetry and bigger $\Delta n$, whereas, in the case of no sneutrino oscillation, the value of $\Delta n$ and the asymmetry should be on the smaller side. This is expected, since, with the cuts that we have imposed, the $\tilde{\nu}_{\tau}$ type of oscillation signal is stronger. When there is oscillation, the splitting between two different charge final states is smaller, and, naturally, the asymmetry is closer to zero.

It is interesting to note that one can also make the processes involving the $\tilde{\nu}_{e / \mu}$ dominate over the process $p p \rightarrow \tilde{\nu}_{\tau} \tilde{\tau}_{1}^{+}$by switching the missing $p_{T}$ cut, i.e., by using $\not \not_{T}>20 \mathrm{GeV}$. In this case, the asymmetry comes out with an opposite sign. By looking at the sign of the asymmetry parameter and with appropriate missing $p_{T}$ cut, one can conclude whether the processes involve predominantly $\tilde{\nu}_{\tau}$ or $\tilde{\nu}_{e / \mu}$. This is another remarkable feature of this study. However, in this case, one gets, in general, a smaller asymmetry because of larger total cross section (which makes the errors smaller though). For $\tan \beta=5,6$, the $\tilde{\nu}_{e / \mu}$ oscillation probability is close to 0.5 . Hence, one would expect close to zero asymmetry for such a parameter point. If there is no oscillation, then the asymmetry is larger, but it never goes to +1 because of the contributions from $\tilde{\chi}_{1}^{0} \tilde{\chi}_{1}^{+}$production.

It is important that, in this study, we have assumed that the staus decay outside the detector. It is also possible that the $R$-parity violating coupling is larger and the staus decay inside the detector after traversing a certain length or they decay promptly. Obviously, the signals discussed here will be changed in those situations, and the analysis to find out the asymmetry will also be different. A detailed discussion on these issues is beyond the scope of this paper, and we hope to come back to these in a future work [7] along with some more details of the present study. One could also look at the production process $p p \rightarrow \tilde{\nu}_{\tau}^{*} \tilde{\tau}_{1}^{-}$. However, the cross section is smaller than in Eq. (3) [8].

In conclusion, we have demonstrated that a suitably defined lepton charge asymmetry may provide us information about sneutrino oscillation at the LHC. This scenario can be realized in an AMSB model with Majorana neutrino masses. Although distinguishing between sneutrino oscillation and no-oscillation is much easier when the SUSY spectrum is known, we have shown that it is also possible to find out the oscillating sneutrino even without the knowledge of the spectrum by looking at simple correlations. One can also provide information about the absolute neutrino mass scale from this study when the SUSY spectrum is known.

We are grateful to D. Choudhury, R. Kinnunen, and S.K. Rai for helpful discussions. This work is supported in part by the Academy of Finland (Project No. 115032). DKG and SR acknowledge the hospitality provided by the Helsinki Institute of Physics. TH thanks the Väisälä Foundation for support.

* Electronic address: dghosh@prl.res.in

$\dagger$ Electronic address: Tuomas.Honkavaara@helsinki.fi

$¥$ Electronic address: Katri.Huitu@helsinki.fi

$\S$ Electronic address: tpsr@iacs.res.in

[1] M. Hirsch, H.V. Klapdor-Kleingrothaus, and S.G. Kovalenko, Phys. Lett. B398, 311 (1997). 
[2] Y. Grossman and H.E. Haber, Phys. Rev. Lett. 78, 3438 (1997).

[3] T. Honkavaara, K. Huitu, and S. Roy, Phys. Rev. D 73, 055011 (2006).

[4] G. F. Giudice, M. A. Luty, H. Murayama and R. Rattazzi, JHEP 9812 (1998) 027 arXiv:hep-ph/9810442 ; L. Randall and R. Sundrum, Nucl. Phys. B 557, 79 (1999) arXiv:hep-th/9810155.

[5] K. Choi, K. Hwang, and W.Y. Song, Phys. Rev. Lett. 88,
141801 (2002).

[6] A. Dedes, H.E. Haber, and J. Rosiek, JHEP 0711, 059 (2007).

[7] D.K. Ghosh, T. Honkavaara, K. Huitu, and S. Roy (work in progress).

[8] W. Beenakker et al., Phys. Rev. Lett. 83, 3780 (1999), (E) ibid, 100, 029901 (2008). 